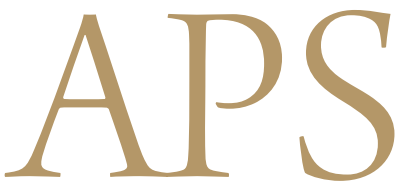

Archives of Plastic Surgery

\title{
The Use of Matriderm and Autologous Skin Graft in the Treatment of Full Thickness Skin Defects
}

\author{
Jang Hwan Min ${ }^{1}$, In Sik Yun ${ }^{1}$, Dae Hyun Lew ${ }^{1}$, Tai Suk Roh ${ }^{2}$, Won Jai Lee ${ }^{1}$ \\ ${ }^{1}$ Department of Plastic and Reconstructive Surgery, Severance Hospital, Yonsei University College of Medicine, Seoul; ${ }^{2}$ Department of Plastic \\ and Reconstructive Surgery, Gangnam Severance Hospital, Yonsei University College of Medicine, Seoul, Korea
}

Background For patients with full thickness skin defects, autologous Split-thickness skin grafts (STSG) are generally regarded as the mainstay of treatment. However, skin grafts have some limitations, including undesirable outcomes resulting from scars, poor elasticity, and limitations in joint movement due to contractures. In this study, we present outcomes of Matriderm grafts used for various skin tissue defects whether it improves on these drawbacks. Methods From January 2010 to March 2012, a retrospective review of patients who had undergone autologous STSG with Matriderm was performed. We assessed graft survival to evaluate the effectiveness of Matriderm. We also evaluated skin quality using a Cutometer, Corneometer, Tewameter, or Mexameter, approximately 12 months after surgery.

Results A total of 31 patients underwent STSG with Matriderm during the study period. The success rate of skin grafting was $96.7 \%$. The elasticity value of the portion on which Matriderm was applied was 0.765 (range, $0.635-0.800$ ), the value of the trans-epidermal water loss (TEWL) was 10.0 (range, 8.15-11.00) $\mathrm{g} / \mathrm{hr} / \mathrm{m}^{2}$, and the humidification value was 24.0 (range, 15.530.0). The levels of erythema and melanin were 352.0 arbitrary unit (AU) (range, 299.25402.75 AU) and 211.0 AU (range, 158.25-297.00 AU), respectively. When comparing the values of elasticity and TEWL of the skin treated with Matriderm to the values of the surrounding skin, there was no statistically significant difference between the groups.

Conclusions The results of this study demonstrate that a dermal substitute (Matriderm) with STSG was adopted stably and with minimal complications. Furthermore, comparing Matriderm grafted skin to normal skin using Cutometer, Matriderm proved valuable in restoring skin elasticity and the skin barrier.

Keywords Skin, artificial / Matriderm / Skin tests
Correspondence: Won Jai Lee Department of Plastic and Reconstructive Surgery, Severance Hospital, Yonsei University Medical College, 50-1 Yonsei-ro, Seodaemoon-gu, Seoul 120-752, Korea

Tel: $+82-2-2228-2210$

Fax: +82-2-361-6947

E-mail: pswjlee@yuhs.ac

Received: 22 Jan 2014 • Revised: 26 Feb $2014 \bullet$ Accepted: 27 Feb 2014

pISSN: 2234-6163 • elSSN: 2234-6171 • http://dx.doi.org/10.5999/aps.2014.41.4.330 • Arch Plast Surg 2014;41:330-336

This study was supported financially by grants from the TRMKOREA.

No potential conflict of interest relevant to this article was reported.

\section{INTRODUCTION}

Skin acts as a barrier to protect the body from the external environment, and it also serves to prevent water loss from the body. When a full-thickness skin defect occurs, a full-thickness skin graft (FTSG) or local flap is typically used for reconstruction.
However, for coverage of a large-sized defect, the dermis can only be reconstructed using a split-thickness skin graft (STSG) rather than a full-thickness skin graft.

Wound healing is a process that proceeds gradually and ultimately aims to recover the skin barrier. This process is complex and involves a variety of cells [1]. The dermis of the damaged 
skin is either replaced by scar tissue that epithelializes to form wound borders, or an STSG is used to close the wound. The amount and arrangement of dermal elastic fibers have great effects on restored skin quality $[2,3]$. However, patchy dermal remnants of the dermal papillae provided by the STSG do not result in a healthy dermal layer for the wound [3]. As a result, STSG provides unsatisfactory results, such as scar contraction and scarring. In this process, reconstruction of the dermis plays an important role in the recovery of wounds, in particular $[1,4,5]$. However, autologous skin grafts have some limitations, which include undesirable functional and cosmetic outcomes that result from hypertrophic scars, poor skin elasticity, and limitations in joint movement due to scar contracture. To complement the drawbacks of STSG, various alternative dermal substitutes, such as acellular dermis or artificial dermal matrix, have been developed and have been applied in the treatment of full-thickness skin defects $[3,6-8]$.

One of the popular dermal substitutes of alternative dermis is acellular dermal matrix. AlloDerm (LifeCell Corp., Branchburg, NJ, USA) is the most widely used acellular dermal matrix. It is a freeze-dried product that is obtained from cadaveric skin and allows improvement in the structure of the dermis. This improvement contributes to wound healing and reduces scar contracture [9-11]. Because this product contains elastin and collagen, it reduces tension and increases the elasticity of the newly formed skin of the restored wound. As a result, contraction of the wound does not occur. As a result, this product improves the functionality and aesthetics of skin grafts [12]. However, there are some limitations to the use of human-derived skin products. Because these are processed from donated human tissue, there could be limits to the supply quantity, leading to an economic burden for disadvantaged patients [13]. Artificial dermis has been widely used to overcome the shortcomings of acellular dermal matrix and to cover full-thickness large skin defects. Artificial dermis reduces scar contraction of the skin graft site and provides skin quality with optimized elasticity and flexibility [6].

Matriderm (MedSkin Solution Dr. Suwelack AG, Billerbeck, Germany) is a highly porous membrane composed of three-dimensionally coupled collagen and elastin. The collagen of the matrix is obtained from the bovine dermis, and the elastin is obtained from the bovine nuchal ligament by hydrolysis. Matriderm provides a scaffold to restore the skin and modulates scar formation. Matriderm reduces the risk of hematoma that occurs after skin grafting due to its hemostatic properties. There are many reports of successful engraftment through a one-step procedure using Matriderm and skin grafting in vitro [14], in vivo [15-18] and in clinical trials [19].

To investigate the usefulness of Matriderm as a sufficient der- mal substitute, we analyzed the survival rate of skin grafts when performed using one-stage grafting with Matriderm and STSG on full-thickness skin defect wounds of various types. We also analyzed the quality of the grafted skin by comparing the elasticity, transdermal water loss, and the erythema and melanin levels of both the adjacent normal skin and the grafted area.

\section{METHODS}

Between January 2010 and March 2012, a retrospective review of patients who had undergone skin grafts with Matriderm for fullthickness skin defects at Severance Hospital was performed. This study was undertaken with permission from the institutional review board of Yonsei University College of Medicine. A total of 31 patients who had undergone skin grafts using Matriderm for full-thickness skin defects were enrolled in this study. We conducted a retrospective chart analysis of patient gender, age, injury mechanism, site, and defect size. The survival rate of the skin graft was defined as the percentage of the entire graft that was considered to be stable and that was well-adhered to the wound bed 7 to 10 days after surgery. The transplantation was rated successful when more than $90 \%$ of the grafted surface area was stable.

In the surgical procedure, the dermal substitute (Matriderm) was applied to the prepared wound bed and soaked in a $0.9 \%$ saline solution after hemostasis of the full-thickness skin defect wound had been achieved. Autologous 1:1.5 meshed skin was grafted over the dermal substitute. The split-thickness skin graft was fixed with staplers or non-absorbable sutures. At the end of surgery, a vacuum-assisted therapy (VAC) device with an intermittent negative pressure of $125 \mathrm{~mm} \mathrm{Hg}$ was applied to prevent the formation of a seroma and to reduce shearing forces on the skin grafts. The VAC device was removed when the dressing was exchanged for the first time on the fourth postoperative day.

\section{Evaluation tools}

To objectively investigate skin quality, we measured four parameters (skin elasticity, humidification, trans-epidermal water loss, and the melanin and erythema levels) and compared the skin quality of the Matriderm and the skin graft area with the adjacent normal skin starting six months after surgery.

The elasticity of the skin was measured using a Cutometer SEM 580 (Courage-Khazaka Electronic GmbH, Cologne, Germany). This device pulled the skin using negative pressure through an $8 \mathrm{~mm}$-diameter probe and measured the elasticity. The degree of elasticity was defined as the maximum value that the skin was distorted by the device. A Corneometer CM 825 (Courage-Khazaka Electronic $\mathrm{GmbH}$ ) evaluated the humidification of the skin by measuring the capacitance of the surface of 
the skin. Trans-epidermal water loss was determined by a Tewameter (Courage-Khazaka Electronic GmbH), which measured the water evaporation. This value indicated the barrier power of the skin. A Mexameter MX 18 (Courage-Khazaka Electronic $\mathrm{GmbH}$ ) was utilized, and a 'photo spectrum analysis' principle was applied to measure the melanin levels and the erythema of the skin. Higher levels of melanin and erythema were represented by darker and redder skin $[11,20,21]$. These objective skin quality values were analyzed with the StatView Package (SPSS Inc., Chicago, IL, USA) using the Wilcoxon signed-rank test. Statistical significance was assumed if a P-value was less than 0.05 . All the results are shown as median (interquartile range).

\section{RESULTS}

A total of 31 patients were grafted with Matriderm and STSG (Table 1). The patients consisted of 20 men and 11 women between the ages of 4 and 68 years. The mean age was $43.32 \pm$ 19.28 years. Causes of the defects included donor site for a fore- arm free flap $(n=16,51.61 \%)$, correction of scar contracture $(\mathrm{n}=4,12.9 \%)$, crushing injury $(\mathrm{n}=4,12.9 \%)$, third-degree burn $(n=2,6.45 \%)$, surgical correction of nevus $(n=2,6.45 \%)$ or keloid ( $n=1,3.2 \%)$, Diabetes mellitus (DM) foot ( $n=1,3.2 \%)$, and osteomyelitis $(\mathrm{n}=1,3.2 \%)$. The size of the defects ranged from 12.5 to $180 \mathrm{~cm}^{2}$, and the mean area was $75.06 \mathrm{~cm}^{2}$. The mean follow-up was 12 months (range, 6-32 months).

Skin grafted with Matriderm using a single-stage procedure was transplanted successfully without infection or inflammatory response in all cases but one, which resulted in a seroma. Although showing a partial loss of $40 \%$ in this one case, the wound was epithelized within two weeks without additional grafting. As a result, the overall survival rate of the matrix and the skin graft in this study group was $96.7 \%$.

The quality of the grafted skin with Matriderm was measured in a total 16 of 31 patients who authorized an examination to evaluate skin quality. To evaluate the skin quality objectively, four measurement items were adopted: 1) skin elasticity, 2) hydration, 3) skin barrier, and 4) darkness and redness using the

\section{Table 1. Patient information}

\begin{tabular}{|c|c|c|c|c|c|c|c|}
\hline No. & Sex & Age (yr) & Cause of defect & Defect area & Defect size $\left(\mathrm{cm}^{2}\right)$ & Graft take & Complication \\
\hline 1 & M & 60 & Osteomyelitis & Lower leg, Lt. & 12.5 & Stable & None \\
\hline 2 & $M$ & 36 & Burn (3rd degree) & 4th, 5th MPJ \& finger, Rt. & 20 & Stable & None \\
\hline 3 & M & 67 & Donor site & Forearm, Lt. & 28 & Stable & None \\
\hline 4 & $\mathrm{~F}$ & 4 & Postoperative scar contracture & Foot, Rt. & 30 & Stable & None \\
\hline 5 & $\mathrm{~F}$ & 44 & Crushing injury & Knee, Lt. & 30 & Stable & None \\
\hline 6 & M & 9 & Postoperative scar contracture & Foot, Lt. & 35 & Stable & None \\
\hline 7 & $\mathrm{~F}$ & 45 & Crushing injury & Inguinal area, Lt. & 35 & Stable & None \\
\hline 8 & $\mathrm{~F}$ & 58 & Donor site & Forearm, Lt. & 40 & Stable & None \\
\hline 9 & $\mathrm{~F}$ & 38 & Donor site & Forearm, Lt. & 45 & Stable & None \\
\hline 10 & $\mathrm{~F}$ & 68 & Donor site & Forearm, Lt. & 48 & Stable & None \\
\hline 11 & $\mathrm{~F}$ & 25 & Donor site & Forearm, Lt. & 50 & Stable & None \\
\hline 12 & M & 51 & Crushing injury & Lower leg, Lt. & 50 & Stable & None \\
\hline 13 & M & 55 & Donor site & Forearm, Lt. & 50 & Stable & None \\
\hline 14 & M & 45 & Donor site & Forearm, Lt. & 60 & Stable & None \\
\hline 15 & M & 54 & Donor site & Forearm, Lt. & 72 & Stable & None \\
\hline 16 & $\mathrm{~F}$ & 24 & Crushing injury & Lower leg, Rt. & 75 & Stable & None \\
\hline 17 & M & 58 & Donor site & Forearm, Lt. & 80 & Stable & None \\
\hline 18 & M & 63 & Donor site & Forearm, Lt. & 80 & Stable & None \\
\hline 19 & M & 54 & Donor site & Forearm, Rt. & 88 & Stable & None \\
\hline 20 & M & 55 & Donor site & Forearm, Lt. & 120 & Stable & None \\
\hline 21 & M & 53 & DM foot & Lower leg, Rt. & 150 & Stable & None \\
\hline 22 & M & 15 & Scar contracture & Forearm, Rt. & 150 & Stable & None \\
\hline 23 & M & 29 & Keloid & Neck & 150 & Stable & None \\
\hline 24 & $\mathrm{~F}$ & 25 & Giant hairy nevus & Forearm, Lt. & 150 & Stable & None \\
\hline 25 & M & 63 & Burn (3rd degree) & Foot, Lt. & 150 & Stable & None \\
\hline 26 & M & 9 & Scar contracture & Upper arm to cubital fossa, Rt. & 171 & Stable & None \\
\hline 27 & $\mathrm{~F}$ & 13 & Giant hairy nevus & Forearm, Rt. & 180 & Unstable (40\%) & Seroma \\
\hline 28 & M & 43 & Donor site & Forearm, Lt. & 63 & Stable & None \\
\hline 29 & M & 63 & Donor site & Forearm, Lt. & 58.5 & Stable & None \\
\hline 30 & M & 54 & Donor site & Forearm, Lt. & 28 & Stable & None \\
\hline 31 & $\mathrm{~F}$ & 63 & Donor site & Forearm, Rt. & 28 & Stable & None \\
\hline
\end{tabular}


Cutometer, Corneometer, Tewameter, and Mexameter, respectively. We analyzed skin conditions by comparing the values between the grafted skin and the surrounding normal skin. The skin elasticity for the Matriderm area was 0.765 (range, 0.635 0.800 ) and for the surrounding normal area was 0.740 (range, $0.665-0.833)(\mathrm{P}=0.518)$. Skin humidification for the Matriderm area was 24.0 (range, 15.5-30.0) and for the surrounding normal area was 36.5 (range, 33.0-41.5), which was significant-

\begin{tabular}{|c|c|c|c|}
\hline Variable & Matriderm area & Adjacent area & P-value \\
\hline Elasticity & $0.765(0.635-0.800)$ & $0.740(0.665-0.833)$ & 0.518 \\
\hline Humidification & $24.0(15.5-30.0)$ & 36.5 (33.0-41.5) & $0.001^{\mathrm{a})}$ \\
\hline TEWL $\left(\mathrm{g} / \mathrm{hr} / \mathrm{m}^{2}\right)$ & $10.0(8.15-11.00)$ & $10.0(8.00-13.00)$ & 0.669 \\
\hline $\begin{array}{l}\text { Melanin value } \\
\text { (AU) }\end{array}$ & $211.0(158.25-297.00)$ & $167.0(143.75-214.75)$ & $0.020^{a)}$ \\
\hline $\begin{array}{l}\text { Erythema value } \\
\text { (AU) }\end{array}$ & $352.0(299.25-402.75)$ & $277.0(212.25-312.25)$ & $0.001^{\mathrm{a})}$ \\
\hline \multicolumn{4}{|c|}{$\begin{array}{l}\text { Values are presented as as median (interquartile range). } \\
T E W L \text {, trans-epidermal water loss; } A U \text {, arbitrary unit. } \\
\text { a) } P<0.05 \text {. }\end{array}$} \\
\hline
\end{tabular}

ly different $(\mathrm{P}=0.001)$. Trans-epidermal water loss for the Matriderm area was $10.0 \mathrm{~g} / \mathrm{hr} / \mathrm{m}^{2}$ (range, $8.15-11.00 \mathrm{~g} / \mathrm{hr} / \mathrm{m}^{2}$ ) and for the surrounding normal area was $10.0 \mathrm{~g} / \mathrm{hr} / \mathrm{m}^{2}$ (range, $\left.8.00-13.00 \mathrm{~g} / \mathrm{hr} / \mathrm{m}^{2}\right)(\mathrm{P}=0.669)$. The melanin level of the Matriderm area was 211.0 arbitrary unit (AU) (range, 158.25297.00 AU), and that of the surrounding normal area was 167.0 AU (range, 143.75-214.75) AU, demonstrating a statistically significant difference $(P=0.020)$. The erythema level of the Matriderm area was 352.0 AU (range, 299.25-402.75 AU), whereas that of the surrounding normal area was 277.0 AU (range, 212.25-312.25 AU) $(\mathrm{P}=0.001)$ (Table 2).

\section{Case 1 (patient no. 9)}

A 38-year-old woman was diagnosed with tongue cancer and received a hemiglossectomy. A tongue reconstruction was performed using a radial forearm free flap, leaving a defect $90 \times 50$ $\mathrm{mm}$ in size (Fig. 1A). A dermal substitute was applied to the defect site (Fig. 1B). After six months, the graft area was stable without any complication and with a good cosmetic outcome (Fig. 1C). On skin analysis, the values for elasticity, humidifica-

\section{Fig. 1. Application of Matriderm on the forearm}

(A) A donor site undergoing radial forearm free flap to reconstruct the tongue. The donor site for the free flap was from a 38-year-old female patient who was diagnoed tongue cancer. (B) The dermal substitute was applied on the donor elevation site. (C) After six months, the graft area was stable without any complications and demonstrated a good cosmetic outcome. On skin analysis, the values for elasticity, humidification, skin barrier and melanin/erythema level of the grafted skin were $0.63,22,9.6 \mathrm{~g} / \mathrm{hr} / \mathrm{m}^{2}$, and 165/356 arbitrary unit, respectively.
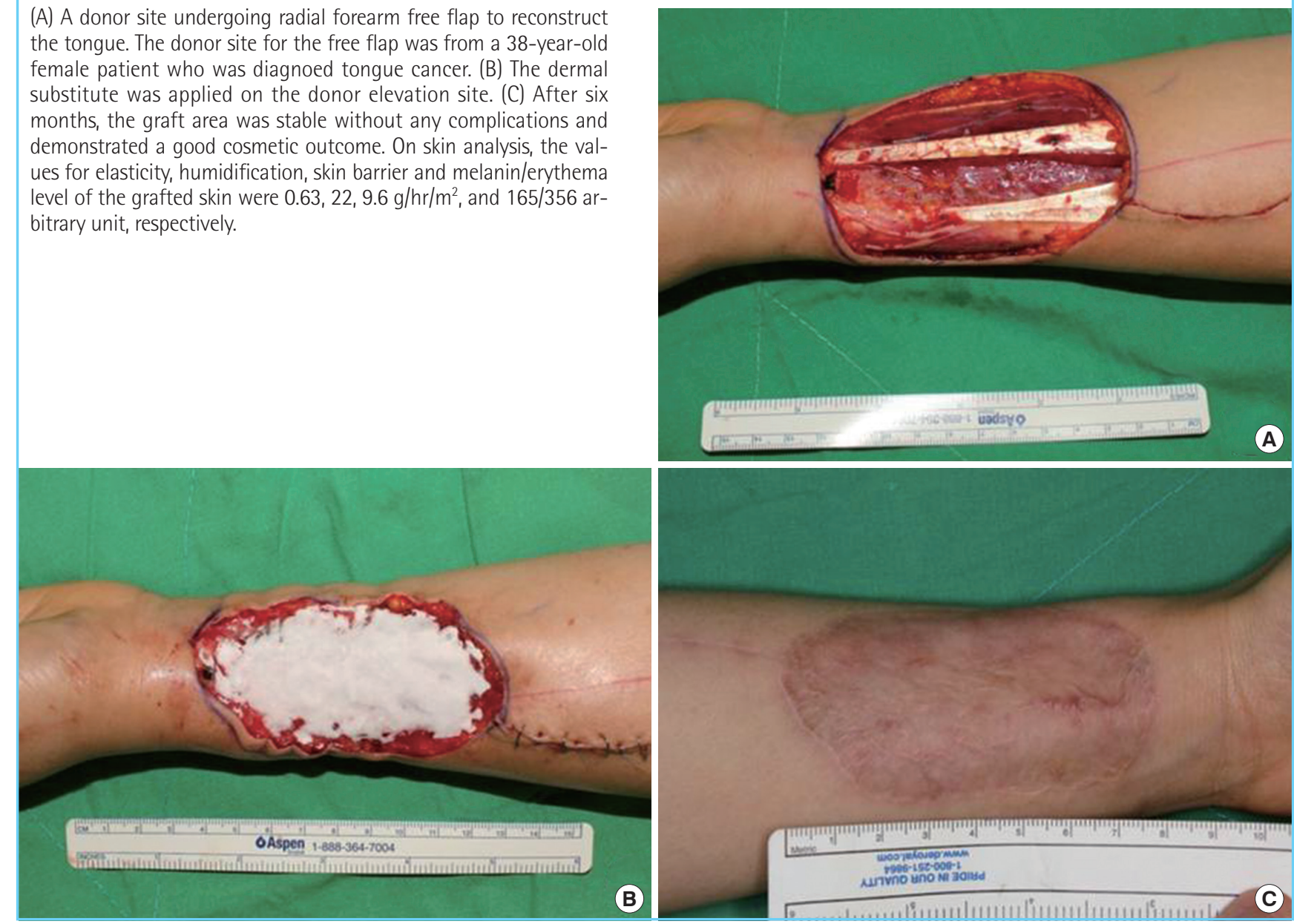


\section{Fig. 2. Application of Matriderm on the foot}

(A) A soft tissue defect of the foot of a 63-year-old male patient who sustained a scalding burn. (B) The dermal substitute was applied to the wound. (C) Postoperative ten months, the incorporation and aesthetic outcome of the graft were good. On skin analysis, the values for elasticity, humidification, skin barrier and melanin/erythema level of the grafted skin were $0.82,29,10 \mathrm{~g} / \mathrm{hr} / \mathrm{m}^{2}$, and 334/405 arbitrary unit, respectively.
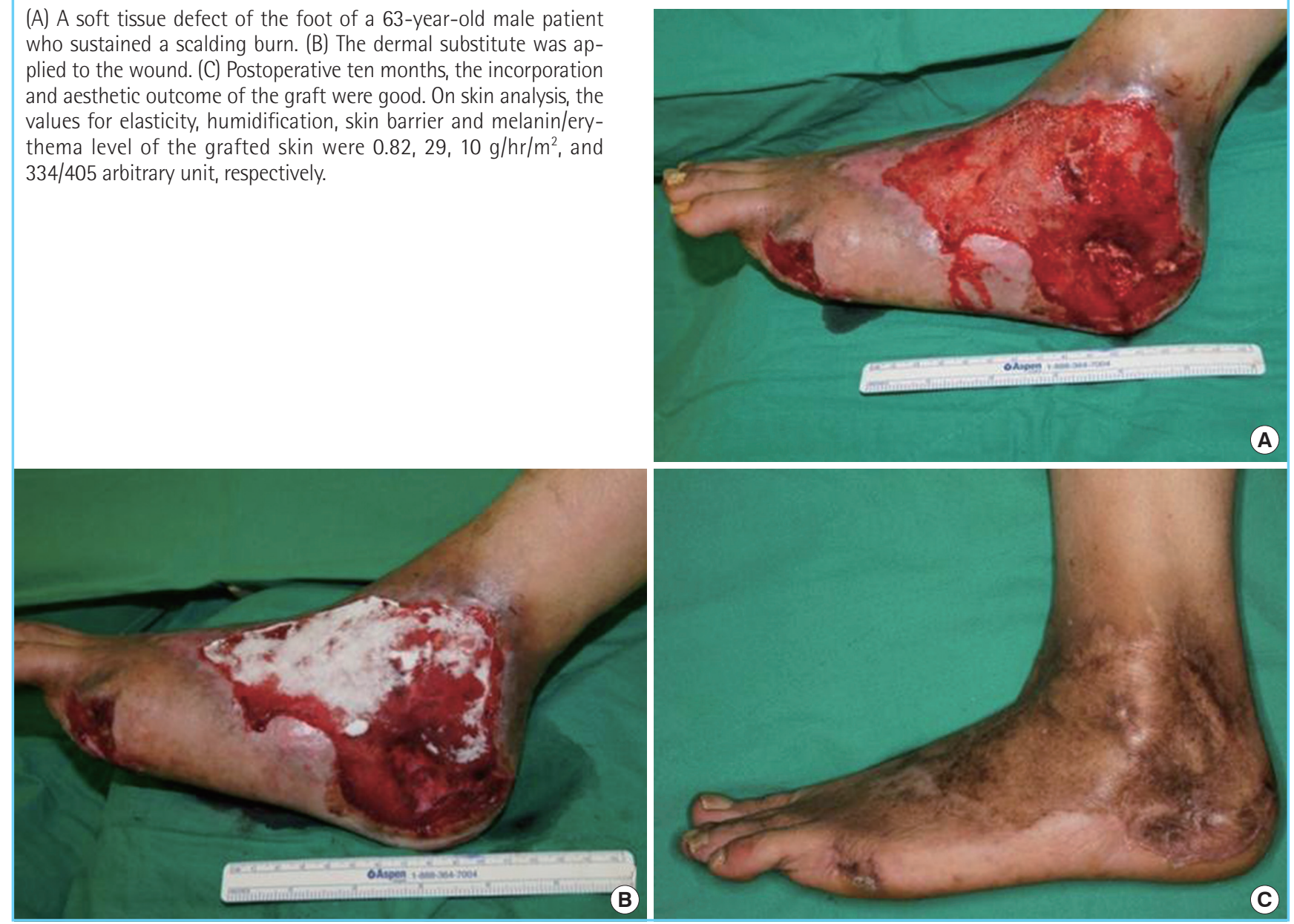

tion, skin barrier and melanin/erythema levels of the grafted skin were $0.63,22,9.6 \mathrm{~g} / \mathrm{hr} / \mathrm{m}^{2}$, and $165 / 356 \mathrm{AU}$, respectively. The values of the elasticity, humidification, skin barrier and melanin/erythema level of the surrounding normal area were $0.77,33,6.8 \mathrm{~g} / \mathrm{hr} / \mathrm{m}^{2}$, and $120 / 214 \mathrm{AU}$, respectively.

\section{Case 2 (patient no. 25)}

A 63-year-old man sustained a third-degree scalding burn involving his left foot (Fig. 2A). After performing debridement, the dermal substitute was applied to the wound (Fig. 2B). Ten months postoperatively, the incorporation of the graft and the aesthetic outcomes were good (Fig. 2C). On skin analysis, the values of the elasticity, humidification, skin barrier and melanin/ erythema levels of the grafted skin were $0.82,29,10 \mathrm{~g} / \mathrm{hr} / \mathrm{m}^{2}$, and 334/405 AU, respectively. The values of the elasticity, humidification, skin barrier and melanin/erythema levels of the surrounding normal area were $0.75,51,9 \mathrm{~g} / \mathrm{hr} / \mathrm{m}^{2}$, and $214 / 351$ AU, respectively.

\section{DISCUSSION}

The purpose of this study was to determine the survival rate of the skin graft in combination with Matriderm and to analyze objectively whether the quality of the grafted skin was of similar quality to the surrounding normal skin via various parameters.

There are several methods for coverage of full-thickness skin defects, such as full-thickness skin grafts or flap transposition. The most reliable methods for reconstruction of large defects are STSG [22]. However, the major limitation of STSG is its undesirable functional and cosmetic results, which are due to hypertrophic scarring, poor skin elasticity, and limitations of joint movement due to scar contractures. In addition, the donor site is insufficient for coverage of large wounds, such as major burns, and morbidity at the donor site is increased. Therefore, alternative dermal matrix has been developed to compensate for the disadvantages of STSG [12,22].

AlloDerm (LifeCell Corp.) is a lyophilized extracellular matrix obtained from cadaveric skin. Its usefulness is widely recognized, but there are drawbacks, such as unstable supply and high 
cost. There are various artificial dermal matrices corresponding to acellular matrices, such as AlloDerm; in this study, the authors used Matriderm. Matriderm is a single laminar matrix consisting of a native (non-cross-linked) collagen matrix supplemented by an elastin hydrolysate. Matriderm is sterilized by gamma irradiation after freeze-drying and is stored at room temperature. In addition, unlike other artificial dermal matrices previously used, successful engraftment using Matriderm and a one-step skin-grafting procedure has been reported in vitro [14], in vivo [15-18] and in clinical trials [19]. In our results, the survival rate was $96.77 \%$, and this result was the same level as a study using an alternative dermal matrix $[1,6]$. These results suggest that Matriderm does not a significant negative influence the survival rate of a skin graft and can be reliably and feasibly used clinically.

The elasticity of the skin with Matriderm was not significantly different from that of surrounding normal skin. This result indicates that grafted skin in combination with Matriderm has an elasticity similar to normal skin. The trans-epidermal water loss was measured using a Tewameter to evaluate the skin barrier. The value of skin barrier where the Matriderm was applied was similar to that of the surrounding normal skin, showing that the Matriderm had a positive effect on skin protection. However, when the degree of skin darkness was assessed by measuring the values of melanin and erythema, the value of the melanin in the Matriderm area was significantly different from that of the surrounding normal skin. In addition, the level of erythema was increased in the Matriderm area, indicating that the Matriderm had a weak effect on decreasing redness. The value of skin humidification was assessed by a Corneomete and also showed a significant difference between the Matriderm area and the normal area, demonstrating the limited effect of Matriderm on improved skin humidification.

Matriderm promotes the regeneration of dermis as a scaffold and consequently decreases scar tissue formation. It also provides optimal dermal wound bed preparation as regenerated skin with extensive formation of rete ridges and capillary loops $[23,24]$. Therefore, the elasticity and skin barrier of the Matriderm area are close to those of the normal surrounding skin.

Recently, many studies of grafted skin have used an inspector's observation of the external appearance and elasticity of the skin. However, these inspections lack objectivity. In addition to the devices mentioned above, many new objective parameters have been introduced $[21,25]$. A long-term study to observe the changes and condition of skin after grafting is needed to provide important research data for improving skin condition. This study demonstrates the role of Matriderm and skin graft in tissue regeneration. A more objective study would include a clear- er distinction between the Matriderm applied area and non-applied area. In treatment of a full thickness wound, Matriderm with an autologous skin graft involves using Matriderm for just a portion of the wound. In this case, when comparing the value of the skin assessment in each area, we would be better able to assess whether the Matriderm had a positive effect on improving graft survival and skin quality.

Matriderm was manageable and did not require an additional procedure during the healing period. The use of this matrix in combination with skin graft resulted in a stable wound and provided promising results in our study. Moreover, the skin elasticity, skin barrier, and skin color of the area over which Matriderm was applied were comparable to those of the adjacent normal skin. Therefore, we consider Matriderm to be a good dermal substitute for full-thickness skin defects.

\section{REFERENCES}

1. Cervelli V, Brinci L, Spallone D, et al. The use of MatriDerm (R) and skin grafting in post-traumatic wounds. Int Wound J 2011;8:400-5.

2. Yannas IV, Burke JF, Gordon PL, et al. Design of an artificial skin. II. Control of chemical composition. J Biomed Mater Res 1980;14:107-32.

3. Juhasz I, Kiss B, Lukacs L, et al. Long-term followup of dermal substitution with acellular dermal implant in burns and postburn scar corrections. Dermatol Res Pract 2010;2010: 210150.

4. Eming SA, Yarmush ML, Morgan JR. Enhanced function of cultured epithelium by genetic modification: Cell-based synthesis and delivery of growth factors. Biotechnol Bioeng 1996;52:15-23.

5. Myers S, Navsaria H, Sanders R, et al. Transplantation of keratinocytes in the treatment of wounds. Am J Surg 1995; 170:75-83.

6. Philandrianos C, Andrac-Meyer L, Mordon S, et al. Comparison of five dermal substitutes in full-thickness skin wound healing in a porcine model. Burns 2012;38:820-9.

7. Burke JF, Yannas IV, Quinby WC Jr, et al. Successful use of a physiologically acceptable artificial skin in the treatment of extensive burn injury. Ann Surg 1981;194:413-28.

8. van Zuijlen PP, van Trier AJ, Vloemans JF, et al. Graft survival and effectiveness of dermal substitution in burns and reconstructive surgery in a one-stage grafting model. Plast Reconstr Surg 2000;106:615-23.

9. Sinha UK, Shih C, Chang K, et al. Use of AlloDerm for coverage of radial forearm free flap donor site. Laryngoscope 2002;112:230-4. 
10. Wainwright DJ. Use of an acellular allograft dermal matrix (AlloDerm) in the management of full-thickness burns. Burns 1995;21:243-8.

11. Yim H, Cho YS, Seo CH, et al. The use of AlloDerm on major burn patients: AlloDerm prevents post-burn joint contracture. Burns 2010;36:322-8.

12. Haslik W, Kamolz LP, Nathschlager G, et al. First experiences with the collagen-elastin matrix Matriderm as a dermal substitute in severe burn injuries of the hand. Burns 2007; 33:364-8.

13. Jones I, Currie L, Martin R. A guide to biological skin substitutes. Br J Plast Surg 2002;55:185-93.

14. Middelkoop E, de Vries HJ, Ruuls L, et al. Adherence, proliferation and collagen turnover by human fibroblasts seeded into different types of collagen sponges. Cell Tissue Res 1995;280:447-53.

15. de Vries HJ, Middelkoop E, Mekkes JR, et al. Dermal regeneration in native non-cross-linked collagen sponges with different extracellular matrix molecules. Wound Repair Regen 1994;2:37-47.

16. De Vries HJ, Mekkes JR, Middelkoop E, et al. Dermal substitutes for full-thickness wounds in a one-stage grafting model. Wound Repair Regen 1993;1:244-52.

17. Enomoto DN, Mekkes JR, Bossuyt PM, et al. Quantification of cutaneous sclerosis with a skin elasticity meter in patients with generalized scleroderma. J Am Acad Dermatol 1996;35:381-7.
18. Janzekovic Z. A new concept in the early excision and immediate grafting of burns. J Trauma 1970;10:1103-8.

19. De Vries HJ, Zeegelaar JE, Middelkoop E, et al. Reduced wound contraction and scar formation in punch biopsy wounds. Native collagen dermal substitutes. A clinical study. Br J Dermatol 1995; 132:690-7.

20. Oliveira GV, Chinkes D, Mitchell C, et al. Objective assessment of burn scar vascularity, erythema, pliability, thickness, and planimetry. Dermatol Surg 2005;31:48-58.

21. van Zuijlen PP, Angeles AP, Kreis RW, et al. Scar assessment tools: implications for current research. Plast Reconstr Surg 2002;109:1108-22.

22. Ryssel H, Gazyakan E, Germann G, et al. The use of MatriDerm in early excision and simultaneous autologous skin grafting in burns: a pilot study. Burns 2008;34:93-7.

23. Hamuy R, Kinoshita N, Yoshimoto H, et al. One-stage, simultaneous skin grafting with artificial dermis and basic fibroblast growth factor successfully improves elasticity with maturation of scar formation. Wound Repair Regen 2013; 21:141-54.

24. Jeon H, Kim J, Yeo H, et al. Treatment of diabetic foot ulcer using matriderm in comparison with a skin graft. Arch Plast Surg 2013;40:403-8.

25. Bloemen MC, van Gerven MS, van der Wal MB, et al. An objective device for measuring surface roughness of skin and scars. J Am Acad Dermatol 2011;64:706-15. 